« Psychologiquement, abstraction faite de son expression par les mots, notre pensée n'est qu'une masse amorphe et indistincte. [...]. Prise en elle-même, la pensée est comme une nébuleuse où rien n'est nécessairement délimité. Il n'y a pas d’idées préétablies, et rien n'est distinct avant l'apparition de la langue. »

« On ne se pénétrera jamais assez de l'essence purement négative, purement différentielle de chacun des éléments du langage auxquels nous accordons précipitamment une existence $[\ldots] »$.

(F. de Saussure) $)^{1}$

«Ce qui m'intéresse, c'est la séparation et l'organisation de ce qui, autrement, se perdrait dans une bouillie originaire. »

Sigmund Freud, Lettre à Lou Andreas-Salomé, 30 juillet 1915².

\title{
Chapitre 9 Derrière le fonctionnement de la RDA en discours : la distinctivité de cinq modes en langue
}

Dans le parcours qui précède, il est apparu que les axes et oppositions selon lesquels la RDA pouvait être différentiellement caractérisée par rapport à l'extérieur des autres champs métadiscursifs - comme mise en jeu de deux actes d'énonciation distincts avec incidence sur le fonctionnement des opérations métalangagières - étaient aussi porteurs de différenciations internes au champ de la RDA - portant sur les types d'articulation, sémantique ou énonciatif, des deux actes d'énonciation (partie II), et sur les opérations métalangagières mises en œuvre (partie III).

Le « croisement » de ces différenciations internes débouche sur une structuration du champ de la RDA en cinq « modes » énonciatifs : définis en termes de traits oppositionnels, comme unités abstraites, situées en deçà de la variété des formes séquentielles observables par lesquelles ils se réalisent et, $a$ fortiori, des effets de sens auxquels ils se prêtent, ces cinq modes sont autant de formes - proposées par la langue - d'appréhender un « discours autre ».

On tentera d'abord (1) de situer cette proposition de structuration par rapport aux clivages théoriques, à forte incidence descriptive, qui traversent les traitements de la RDA, notamment quant au rapport entre le champ de la RDA - défi-

1 Respectivement F. de Saussure, Cours de linguistique générale, éd. critique T. de Mauro (1916 : 155) et Écrits de linguistique générale (2002 : 64-65).

2 Lou Andreas-Salomé, Correspondance avec Sigmund Freud, Gallimard, 1979, p. 44, cité par M. Schneider (2010 : 129).

ว Open Access. (C) 2020 Jacqueline Authier-Revuz, published by De Gruyter. (cc))BY-NC-ND This work is licensed under the Creative Commons Attribution-NonCommercial-NoDerivatives 4.0 License. https://doi.org/10.1515/9783110641226-016 
nissable au plan sémantico-référentiel et observable dans son fonctionnement multiforme en discours - et l'ordre de la langue, comme système de différences formelles ; puis, après avoir présenté (2) cette structuration, on évoquera (3), en forme de "programme » pour la description de chacun des modes et de leur fonctionnement en discours - qui serait l'objet d'un autre ouvrage -, quelques axes et problématiques sur lesquels elle ouvre.

\section{La RDA dans la langue}

\subsection{Champ sémantico-référentiel et dispersion des formes observables}

La RDA, champ défini par son référent - un acte d'énonciation distinct de l'énonciation en cours -, est instituée comme secteur métalangagier à dimensions sémantique (la représentation d'un acte d'énonciation porteur de sens) et énonciative (la mise en jeu de deux ancrages distincts) : ce champ ne correspond pas dans la langue à un sous-système de morphèmes, d'oppositions paradigmatiques propres, tels qu'en présentent la déclinaison, le système des temps verbaux, ou la diathèse actif/passif, par exemple. La « réalisation » formelle de la RDA recourt à l'ensemble le plus disparate qui soit (quant aux plans dont ils relèvent) d'éléments qui ne lui sont pas propres ; l'identification d'une « forme de RDA » est ainsi susceptible de passer par des éléments, diversement combinés, relevant : du statut sémiotique (autonymie), de la syntaxe phrastique et interphrastique (relations de rection, incidence, anaphore... ; fonctions d'objet direct, apposition, circonstants...), du lexique (métadiscours de la catégorisation), des marques d'ancrage énonciatif (référentielles et modales), de la ponctuation au sens large (guillemets, tirets, alinéas, caractères, disposition paginale...), de la prosodie (variations de hauteur, pauses démarcatives...), du plan sémantico-discursif (cohérence entre une " annonce » de dire et son explicitation, concordance/discordance au plan des contenus ou des manières de dire, entre une forme de RDA et son co-texte)...

Dans la multiplication des éléments hétérogènes qui y jouent, la dispersion des formes de RDA n'est pas réductible à l'organisation paradigmatique de quelques « patrons » morpho-syntaxiques.

\subsection{Le mirage paradigmatique de la vulgate}

Méconnaissant cette dispersion, c'est pourtant par trois « patrons » morpho-syntaxiques, DD-DI-DIL, constitués en paradigme du « discours rapporté », qu'une 
vulgate encore insistante aujourd'hui, représente ce champ ${ }^{3}$. Le jeu différentiel à l'intérieur de la RDA se trouve ramené à une opposition grammaticale, au sens étroit (morpho-syntaxique) du terme ${ }^{4}$ : sur le mode où on peut coupler, par exemple, les structures morpho-syntaxiques de l'actif et du passif :
(1) (a) $\mathrm{SN}_{1} \mathrm{VSN}_{2}$
et (b) $\mathrm{SN}_{2}$ être Vppé par $\mathrm{SN}_{1}$.
Le chien surveille le troupeau.
Le troupeau est surveillé par le chien.

on va - abusivement - coupler les patrons morpho-syntaxiques « du DD » et « du $\mathrm{DI} »$ :
(2) (a) $l$ dire : « $\mathrm{p} »$
et
(b) $\quad l$ dire que $\mathrm{p}$
Il dit : « je viens ».
Il dit qu'il vient.

et, de la même façon que l'on dit que (1b) est la forme du passif français correspondant à l'actif (1a), dire que (2b) est la forme du DI, correspondant au DD (2a).

Cette approche, appréhendant la différence entre les deux modes du DD et du DI par l'opposition paradigmatique de deux types « séquentiels », est à l'évidence inadéquate : l'extrême diversité des formes sous lesquelles se réalise chacun des deux modes est irréductible à ce « formatage » qui promeut une réalisation particulière comme la forme du DD ou du $\mathrm{DI}^{5}$. Cette restriction à une structure morpho-syntaxique de ce qui est un champ de réalisations formelles différentes, trouve son pendant au plan sémantique quand, par exemple, on caractérise le DD par la restitution textuelle à laquelle pareillement on ne saurait réduire (on l'a vu, chap. 8.2.2.2 p. 263) l'éventail des effets de sens d'un DD « en emploi et en action ».

Dès lors que l'on se confronte à l'extrême variété observable des réalisations de la RDA - explorée par nombre d'études de corpus ces vingt dernières années -, au plan formel comme au plan du sens en discours, le cadre de la vulgate avec ses

3 Rappelons-en schématiquement les principaux « articles » : trois formes DD-DI-DIL, la première caractérisée par sa « simplicité » syntaxique et sa « fidélité » de reproduction, la seconde, obtenue par subordination (et « transposition ») à partir de la première, la troisième, donnée comme DI « allégé » de la subordination et/ou «mélange » de DD et de DI, et caractérisée comme spécifiquement littéraire.

$4 \mathrm{Si}$, en revanche, par « Grammaire » d'une langue on désigne l'intégralité de ce qui - tous niveaux compris - la constitue en « système de différences », alors l'opposition, par exemple, entre les deux « modes » du DD et du DI au niveau abstrait où elle se situe (cf. ci-dessous), est un fait de langue, et relève bien de sa « Grammaire ».

5 Le parcours de G. Komur (2003 : 32-38) tend à faire apparaître le caractère majoritairement non nécessaire (non définitoire) des traits classiquement proposés par les grammaires comme caractéristiques « des trois modes». 
trois « patrons » morpho-syntaxiques, assortis de la textualité ${ }^{6} \mathrm{du}$ DD, ne peut que craquer... Mais le dépassement des « insuffisances de la vulgate ${ }^{7}$ peut emprunter des chemins divers : le consensus quant à la nécessité, une fois élargi le champ des données, de prendre en compte des énoncés incompatibles - au plan de la forme ou du sens - avec le « pseudo-paradigme » traditionnellement mis en avant, peut se rompre, clivé, crucialement, par la reconnaissance ou non d'un double plan, celui de la langue, comme système abstrait de distinctivité, et celui auquel il s'articule, de la discursivité observable ; à titre d'alternative au paradigme disqualifié (trio DD-DI-DIL ou couple DD-DI), des traitements radicalement différents de l'ensemble du champ proposent, respectivement, correspondant à des choix théoriques opposés, une issue vers l'au-delà d'une mixité-continuum en discours (1.3) vs l'en deçà d'une structuration différentielle en traits pertinents de langue (1.4).

\subsection{L’au-delà, discursif, de la mixité-continuum}

La question de l'intégration, dans la description du champ de la RDA, des formes qui en étaient exclues par l'étroitesse, morpho-syntaxique autant que sémantique, de la caractérisation classique des «types » du " paradigme », peut se résoudre dans un traitement en termes de continuum : faisant place, entre les types fixés par la tradition, à une multiplicité de faits de mixité, hybrid(is)ation, mélange qui, excédant les premiers, sont cependant caractérisés à partir de ceux-ci, il congédie, de fait, le principe même d'un système d'oppositions structurant, en langue, la variété des réalisations en discours.

À plusieurs reprises, sur des points particuliers ${ }^{8}$, nous avons évoqué cette démarche, en opposition au "parcours de distinctivité » tenté ici : présente, notamment, chez M.-M. de Gaulmyn (1983) identifiant une pluralité de types « mixtes » entre DD et DI par lesquels se trouve « abattu le mur rigide qui [les] séparait » (p. 324), ou dans le traitement de la « citation » par U. Tuomarla (2000) qui repose sur des « continuums », « hybridisation DD/DI », « cas intermédiaires » «brouillant les deux catégories DD et DI », cette démarche soutient le parcours d'ensemble du discours rapporté proposé par L. Rosier (1999) : la confrontation systématique du « couple binaire » des « catégories canoniques

$6 \mathrm{Au}$ sens de fidélité littérale de la reproduction (cf. chap. 8.2.2.2, p. 263).

7 Que je notais dans Authier-Revuz (1992a : 38).

8 Cf., par exemple, chap. 8 Remarque 3, p. 271 et Remarque 5, p. 275. 
du discours rapporté grammaticalisé $»^{9}, \mathrm{DD} / \mathrm{DI}$, tel que la tradition en assure la prégnance ${ }^{10}$, avec tous les cas qui, au plan syntaxique, typographique ou sémantique, "ne rentrent pas » dans ce carcan, y débouche sur la notion de mixité. L'identification comme forme hybride ou mixte - c'est-à-dire, à partir des catégories reçues du DD/DI, comme «empruntant » aux deux types de discours, les " confondant » ou les " mélangeant » au sein d'une unité phrastique ${ }^{11}$ - d'une série de faits aussi divers que le DIL, le DI « avec guillemets », le DD « avec que », le DD paraphrastique, l'incise de DI... a pour corollaire la « mise à mal » de la « dichotomie DD/DI », dans un " affaiblissement » des frontières « entre DD et DI », un « décloisonnement » ou un « rapprochement » des deux types, « empiétant sur le terrain de l'autre $»^{12}$. La visée explicitée d'une « organisation générale des discours rapportés sous la forme d'un continuum » qui, " réalisé en discours " ${ }^{13}$, est mis au jour à travers la " pratique », l'usage et « les effets produits par l'emploi de telle ou telle forme $»^{14}$, comme traversé « par un mouvement d'appropriation du discours d'autrui par le sujet, de la mise à distance maxi-

9 Cf. les repères intéressants placés par L. Rosier (1999 : 9, 13-20) dans le processus menant des approches narratives et rhétoriques de la mimesis/diegesis et de l'oratio recta/obliqua, à la conception grammaticalisée du couple DD/DI.

10 C'est en effet à partir des catégories telles que la tradition les propose - "dogmatiques », "sclérosées », mais chargées d'un poids idéologique dans les images qu'en ont les énonciateurs que la description, critique, de L. Rosier (1999), est organisée, aux plans morpho-syntaxique (a) et sémantique (b) : (a) « Le discours direct sera symbolisé par la forme dire : " ... », le discours indirect par la forme dire que ». (1999: 40), (b) « [...] un DD citant les mots des autres et un DI traduisant les mots des autres [...]» (ibid. : 114).

11 Cf. par exemple : « [...] la pratique actuelle du DR [...] mélange les critères distinctifs des deux formes cloisonnées. (1999: 82) Une forme « mixte » [...] confronte un type de discours (le DD) avec la caractéristique principale de l'autre (le que du DI). (1999 : 88) Une forme mixte emprunte à l'un ou à l'autre des discours des marqueurs qui lui sont spécifiques et/ou des effets de sens particuliers. (1999 : 202, gdt) ». Le traitement proposé apparaît ainsi, curieusement, dépendant des catégories contestées, le détour par leur image réfractée chez les énonciateurs ne le mettant pas à l'abri de la critique adressée par l'auteur (1999 : 97) aux théories qui « restent dans leur majorité tributaires » de la saisie grammaticale canonique des formes de DR.

12 Cf. par exemple : l'existence d'un DD paraphrastique « plaide pour un rapprochement DD/ DI » (1999 : 114) ; celle de l'incise en DI « apparaît comme une imbrication des différents discours, qui affaiblit les frontières entre DI et DD » (1999: 262), et permet « de décloisonner les DD et DI » (1999 : 265) ; et, de façon générale, est indiquée une « volonté de montrer l'affaiblissement des frontières entre DD et DI ( 1999 : 246), et dans « l'usage des discours rapportés [dans le corpus dépouillé] une tendance significative à empiéter sur le terrain de l'autre » $(1999$ : 202).

13 Respectivement (1999: 125, gdt), (1999 : 137 idt).

14 (1999 : 24), tels, par exemple, « l'effet de fidélité attaché au DD » (1999 : 245) ou les « effets de DD » de « l'incise, [qui] "tire” le discours [indirect] dans lequel elle s'insère vers le direct. » (1999: 261). 
male à l'ingestion/digestion de la parole d'autrui » (1999 : 9) marque, nettement, le changement de terrain qui accompagne la contestation d'une opposition formelle inadéquate : il ne s'agit pas dans cette approche de mettre au jour, se substituant à l'opposition mise en défaut, une autre structuration différentielle, mais de déplacer l'appréhension du discours rapporté du côté d'une discursivité intégrant - en y « diluant » la langue - psychologie, idéologie, sens... ${ }^{15}$ La mise en cause de la partition canonique conduit ici à rejeter le principe même d'une partition en langue.

\subsection{L'en deçà d'une distinctivité abstraite, en langue}

Posant, à propos de DD précédés de que, la question de l'intégration au champ de la RDA de formes mettant en défaut le paradigme établi, Bruña-Cuevas (1996) ${ }^{16}$ récuse la solution offerte par des catégories comme "discours mixte, discours hybride et mélange de SI et de SD » (Rosier, 1999 : 244) :

Ces dénominations ne nous semblent pas adéquates, elles nous semblent plutôt révéler l'embarras devant une forme qui échappe aux typologies traditionnelles du DR, dès lors manifestement mal établies. (p. 30)

Ainsi, Benveniste (1965), au seuil d'une réanalyse linguistique du système des pronoms du français, dénonçait-il dans des propositions descriptives récentes la permanence d'une « conception, stylistique ou impressive » propre à « seulement déguiser en notion psychologique une réalité linguistique insuffisamment décrite. » (1965: 198).

C'est dans ce sens que s'inscrit la démarche que je tente de suivre : celle - à l'opposé d'un continuum déployant une variété d'énoncés soustraite au jeu contraignant des oppositions - d'une restructuration différentielle du champ,

15 Le déplacement vers la «mixité » et le continuum apparaît, pour L. Rosier (1999: 107), comme le corrélat d' « une linguistique qui ne peut plus, depuis une trentaine d'années, ignorer le sujet, le sens » ; tandis que chez D. Torck (2004 : 246) il est envisagé comme relevant d'un déplacement disciplinaire, hors de la linguistique : ainsi l'analyse proche («frontières formelles [tendant] à s'effacer »; « formes mixtes qui abondent »; « difficile et [...] improductif de figer les différentes formes de DR dans des valeurs canoniques ») est-elle formulée dans une perspective explicitement interactionnelle, visant les enjeux relationnels du fonctionnement des séquences de DR, que l'auteur caractérise comme se situant « probablement plus près de certaines approches du DR en psychologie sociale et en anthropologie linguistique [...] que d'approches linguistiques ». 16 Auquel L. Rosier (1999 : 88, 244) fait place en contrepoint - de désaccord - avec sa propre démarche. 
telle que les frontières ainsi retracées permettent de rendre compte de l'ensemble des formes et de leur valeur.

L. Rosier signale cette divergence d'approche, s'interrogeant sur le fait que la reconnaissance, effective, dans certains travaux ${ }^{17}$, de faits excédant les définitions traditionnelles des types, telle que « on pourrait s'attendre à une prise en compte de la notion de mixité formelle ${ }^{18}$, n'y conduise pourtant pas leurs auteurs, obstinément attachés à une structure différentielle...

À travers l'exemple, rencontré chap. 8, du DD passant, à l'égal d'un DI, par l'opération paraphrastique, il convient de souligner que le maintien, face à des formes questionnant la définition canonique des types, d'un rapport d'opposition ne signifie pas la conservation fétichiste d'un paradigme que démentent les faits, mais passe par une redéfinition des types et des frontières selon lesquelles ceux-ci s'opposent : il est clair qu'une opposition DD/DI où le DD est défini comme « textuel » est « mise à mal » par l'existence des DD paraphrastiques ; mais dès lors que le DD est redéfini par le trait « autonyme » (situé « en deçà » de la propriété de " textualité », qui n'est qu'une des valeurs qu'il peut prendre en discours), une opposition DD/DI demeure, refondée sur une frontière située ailleurs, en deçà, à un plan - en langue - de plus grande abstraction. De la même façon, le trait « autonyme » par lequel le DD s'oppose - en combinaison avec d'autres traits - au DI, s'il comporte l'implication formelle d'une rupture syntactico-sémiotique de l'élément autonyme par rapport à son environnement phrastique, se situe également en deçà de toute réalisation séquentielle particulière, telle que, par exemple, la forme $l$ dit : « ... ", hypostasiée comme « le discours direct ».

Ainsi, à un DD défini (3) positivement par un « patron » linéaire et une valeur

(3) -1 dit : « ...

- textualité

on substituera un « mode $\mathrm{DD}$ » défini différentiellement dans le champ de la RDA par un faisceau de traits distinctifs $(4)^{19}$ :

17 Rey-Debove et Authier notamment, $c f$. Rosier (1999 : 108-114) par exemple : « Dans ce cadre [métalinguistique, J. Authier] a touché aux formes mixtes que nous avons isolées plus haut. Nous verrons comment elle les intègre dans une conception renouvelée de l'approche classique du DR ». (1999 : 108)

18 Rosier (1999: 112, gdt).

$19 \mathrm{Cf}$. « aucun fait de langue [...] n'existe un seul instant par lui-même hors de son opposition avec d'autres » (Saussure, 2002 : 66). 
(4) - discours autre objet (vs source)

- « $\boldsymbol{e} »$ avec autonymie (vs sans)

- dualité d'ancrages énonciatifs ( $v s$ unité ou division)

qui, en deçà de toute linéarisation et de toute valeur en discours, est susceptible de se réaliser, parmi d'autres, sous la forme ou avec le sens noté en (3).

\subsection{L'enjeu du « réel de la langue »}

L'inadéquation du paradigme des "types » tels que fixés par la vulgate et de la partition qu'elle opère dans le champ de la RDA n'est pas contingente : un simple déplacement des lignes d'opposition aux plans où elles sont tracées - types séquentiels ou sens en discours - ne peut être une issue ; la " dispersion » des formes relevant de la RDA est irréductible à un quelconque paradigme opposant des types séquentiels : la description doit changer de niveau. Et c'est bien ce qu'effectuent, dans des directions opposées, les deux approches envisagées : là où le mouvement de remise en cause des oppositions " canoniques " se fait, pour l'une - en rejetant le principe même d'oppositions en langue - vers l'aval d'un continuum d'observables en discours, l'autre, que nous suivrons, se fait vers l'amont d'une structuration différentielle en traits pertinents relevant, en langue, d'un plan de plus grande abstraction que celui des types grammaticaux et - a fortiori - des effets de sens de leurs occurrences en discours.

Il est clair que cette divergence d'approche s'inscrit dans un clivage dépassant largement la question du traitement de la RDA : le congédiement de la langue, au sens saussurien du terme - comme système abstrait de distinctivité -, volontiers dénoncée comme artefact ou réduite à une « norme » restrictive, au profit de catégories psychologiques, argumentatives, communicationnelles... opérant au plan du discours, est un mouvement de fond qui, traversant depuis trois décennies l'ensemble des $"$ sciences du langage $»^{20}$, affecte spécifiquement les secteurs reconnus comme impliquant énonciation, subjectivité,

20 Dès L'Amour de la langue (1978), Milner s'interroge de façon aigüe sur ce qui, dans la linguistique - « s'attacher à la langue comme telle, y reconnaître les facettes d'un réel »-, « importune », au point que « se développe incessamment » ce qu'il appelle une " anti-linguistique » (p. 124-126). À cette analyse Pêcheux (1982) fait écho, dénonçant « l'aversion [...] à l'égard du propre de la langue » et le mouvement conduisant à « Penser contre Saussure ». Deux décennies plus tard, se retournant sur Le Périple structural, Milner (2002) radicalise son constat : « Depuis 1973, la restauration du substantiel au détriment de toute forme (des significations au détriment 
sens... Contre l'apparente " évidence » d'une alternative langue ou énonciation/discours, je revendique, dans la ligne de Benveniste - et de la « double signifiance $»^{21}$ par laquelle il articule négativité abstraite du signe et positivité concrète des énoncés - la reconnaissance du " réel de la langue » et de son abstraction différentielle, au principe de l'approche des faits d'énonciation et de discours ${ }^{22}$.

\section{Structuration différentielle - en langue - du champ de la RDA}

\subsection{Trois oppositions pertinentes}

Le parcours qui précède a fait apparaître trois oppositions pertinentes, opérant des distinctions dans le champ des énoncés observables en RDA :

A. celle (ch. 3 schéma VII p. 100) du statut sémantique donné, dans l'énoncé, au discours autre qui y est représenté comme

\section{1. objet du dire}

glosable en $\mathrm{A}_{1}$ : ce dont on parle ; parler de opposition qui partage :

$\mathrm{A}_{1}$ : Réponse de Jean : « Je refuse » Il a dit son désaccord.

\section{2. source du dire}

vs $\mathrm{A}_{2}$ : ce d'après quoi on parle ; parler d'après

vs $\mathrm{A}_{2}$ : D'après lui, j’ai fait ce qu'il fallait.

«J'ai assuré », comme dirait Marie.

de tout signifiant) n'a pas cessé d'accentuer ses effets. [...] Qu'il s’agisse des données, des méthodes, des notions, Saussure n’a pas existé. » (p. 239).

21 Dont l'ouverture, « dépassant » le signe saussurien en direction du domaine de « la langue en emploi et en action » (le " sémantique ») ne cède à aucun moment sur cela (le « sémiotique ») à quoi s'articule « à la base » le fonctionnement langagier : le système des oppositions distinctives dont le jeu délimite, définit, institue les unités de langue. (cf. particulièrement : « La forme et le sens dans le langage » et « Sémiologie de la langue » in Benveniste (1974).

22 Sur cette position (qui relève du « courant énonciatif au sens étroit » ou « néo-structuraliste » que C. Fuchs (1981) repère, en évoquant les noms de Bally, Benveniste, Guillaume, Culioli, comme « [partant] des formes de la langue »), voir Authier-Revuz (1995/2012 : 61-71) « Hétérogénéité du champ énonciatif - Du linguistique affecté par des extérieurs » « Penser “avec Saussure” pour saisir l'énonciation », et (1995/2012 : 469-473) « La langue comme ordre propre », « Un réel méconnu dans des "dépassements" du structuralisme». 
B. celle (ch. 8) du statut sémiotique de la représentation de $\boldsymbol{e}$ en $\mathbf{E}$ : ordinaire, transparent, faisant usage des mots $v s$ avec autonymisation, monstration des mots, blocage de synonymie :

\section{1. ordinaire (en usage)}

\section{2. avec autonymisation}

opposition qui partage :

$\mathrm{B}_{1}$. D’après lui, j’ai fait ce qu'il fallait. $\quad \mathrm{B}_{2}$ « J'ai assuré », comme dirait Marie.

Il a dit son désaccord

Réponse de Jean : « Je refuse ».

Jean $_{\mathrm{i}}$ se fâche : Est-ce qu'on $\mathrm{le}_{\mathrm{i}}$ croit naîf ?

Le croisement des deux oppositions opère une quadripartition :

\begin{tabular}{|c|c|c|}
\hline $\begin{array}{l}\text { A } \\
B\end{array}$ & $A_{1}$ : DA objet du dire & $A_{2}:$ DA source du dire \\
\hline $\mathrm{B}_{1}$ : sémiotique ordinaire & $\begin{array}{l}{\left[A_{1} B_{1}\right]} \\
\text { ex. : Jean a dit son désaccord. } \\
\text { DI }\end{array}$ & $\begin{array}{l}{\left[A_{2} B_{1}\right]} \\
\text { ex. : D'après Jean, j'ai fait ce } \\
\text { qu'il fallait. } \\
\text { MAS }\end{array}$ \\
\hline $\mathrm{B}_{2}$ : avec autonymisation & $\begin{array}{l}{\left[\mathrm{A}_{1} \mathrm{~B}_{2}\right]} \\
\text { ex. : Réponse de Jean : «Je } \\
\text { refuse. » } \\
\text { DD } \\
\text { ex. : Jean }{ }_{\mathrm{i}} \text { se fâche : Est-ce } \\
\text { qu'on le } \text { croit naïf ? }^{\text {Bivocal }}\end{array}$ & $\begin{array}{l}{\left[\mathrm{A}_{2} \mathrm{~B}_{2}\right]} \\
\text { ex. : Jean a « assuré » comme } \\
\text { dirai Marie } \\
\\
\text { MAE }\end{array}$ \\
\hline
\end{tabular}

C. celle (chap. 4, p. 145) portant sur le type d'articulation en $\mathbf{E}$ des ancrages énonciatifs des deux actes $\mathbf{A}$ et $\boldsymbol{a}$ : opposition ternaire, contrairement aux deux autres, elle fait passer une différenciation supplémentaire à l'intérieur de la partition effectuée par les traits A et B - entre unité énonciative en $\mathbf{A}$, dualité énonciative en $\mathbf{A}$ et en $\boldsymbol{a}$ - et elle dégage, « excentré », le type autre de la division des ancrages primaires entre A et $\boldsymbol{a}$ propre au Bivocal : 
1 ancrage unifié en $A$ deux ancrages distincts en $A$ et en $a$

3 ancrage partagé entre $A$ et $a$

opposition qui partage :

\begin{tabular}{|c|c|c|}
\hline$C_{1}$ : ancrage unifié en $\mathbf{A}$ & $\begin{array}{l}C_{2}: 2 \text { ancrages distincts en } \\
\text { A et } \boldsymbol{a}\end{array}$ & $\begin{array}{l}C_{3} \text { : ancrage partagé entre } \\
\text { A et } a\end{array}$ \\
\hline $\begin{array}{l}{\left[A_{1} B_{1}\right]} \\
\text { ex. Jean a dit son désaccord. } \\
{\left[A_{2} B_{1}\right]} \\
\text { ex. D'après Jean, j'ai fait ce } \\
\text { qu'il } \\
\text { fallait. } \\
{\left[A_{2} B_{2}\right]} \\
\text { ex. « J'ai assuré », comme } \\
\text { dirait Marie. MAS } \\
\quad \text { MAE }\end{array}$ & $\begin{array}{l}{\left[A_{1} B_{2}\right]} \\
\text { ex. : Réponse de Jean : «Je ref } \\
\text { use. " }\end{array}$ & $\begin{array}{l}{\left[A_{1} B_{2}\right]} \\
\text { ex. Jean } \text { se fâche : est-ce } \\
\text { qu'on le } e_{i} \text { croit naïf ?. }\end{array}$ \\
\hline
\end{tabular}

\subsection{Cinq « MODES » de RDA}

\subsubsection{Une analyse sur plusieurs plans}

C'est par la mise en regard d'énoncés concrets de RDA - attestés ou fabriqués que sont dégagés les traits $\mathrm{A}, \mathrm{B}, \mathrm{C}$, en fonction desquels ils s'opposent, traits relevant, abstraits, généraux, du plan de la langue comme système de différences ; de leur combinatoire émergent cinq combinaisons ou "formules » de traits - sémantique, sémiotique, énonciatif - structurant différentiellement, en langue, le champ de la RDA.

Ces combinaisons de traits se situent en deçà de la diversité des types formels selon lesquels peut se réaliser leur linéarisation - les formes offertes par la langue pour la représentation du discours autre relevant de deux « étages » (non linéaire et linéaire) articulés de son système.

Chacun des cinq modes de RDA se déploie donc sur trois plans : celui (I) d'un ensemble fini de traits différentiels, abstraits et pauvres sémantiquement, ouvrant (II), au plan de la linéarité, sur la « zone » de formes par lesquelles il se réalise, large espace de variation où jouent les niveaux morpho-syntaxique, lexical, typographique, sémantique, discursif, dans lequel la description peut repérer, non pas un système différentiel structurable en paradigme, mais des types d'agencements linéaires qui débouchent (III), en discours, sur des occurrences concrètes et leurs effets de sens singuliers, dont l'infinité et le continuum - certes «balisables » en termes génériques, stylistiques, idéologiques... - excède radicalement la systématicité différentielle (du I) qui les « porte ». 
En changeant de plan d'analyse, des traits différentiels (I) aux types d'agencements linéaires (II), on ne quitte pas l'espace du « répétable » et du « discontinu » - qui est celui des formes de langue ; c'est avec le plan (III) des événements de parole, où la langue, " en emploi et en action », s'articule au " monde » qu'on aborde, au-delà de la négativité structurée de signes, à la positivité ininventoriable des sens.

Pour reprendre le cas, évoqué ci-dessus, du MODE DD, on distinguera ainsi:

(I) : sa Formule, combinant par les trois traits $\left[\mathbf{A}_{1}-\mathbf{B}_{2}-\mathbf{C}_{2}\right]$, i.e. parler du DA (vs parler d'après), avec autonymie (vs sans), avec dualité énonciative (vs unité ou partage) ;

(II) : l'espace des formes par lesquelles il peut se réaliser, où figurent des types linguistiques séquentiels illustrés ici, par commodité, à l'écrit et à travers une réalisation lexicale comme :

(5) (a) Il a dit : « Je suis heureux. » (b) Sa déclaration (je suis heureux) a surpris. (c) Je suis heureux, proclame-t-il. (d) « Je suis heureux. » Ces mots ont surpris. (e) Il se réjouit : « Je suis heureux. » (f) Il arrive. Je suis heureux. Il repart. Etc.

(III) : le déploiement des effets de sens dans les occurrences particulières, où à la même forme au plan (II) : Il a dit : " Je suis heureux ", pourront être associés, en contexte, des sens caractérisables (encore en deçà de leur stricte singularité) par des effets de théâtralité, distanciation, identification, textualité, etc.

Relativement à cet étagement, dans lequel c'est au plan (I), des traits distinctifs, que se définit le MODE, les « définitions » du DD comme forme dire : «... » ou discours rapporté « textuel » relèvent d'une illégitime " promotion » au rang de propriété distinctive de langue, de ce qui n'est qu'une des formes de l'espace (II), ou qu'un des effets de sens du niveau discursif (III).

\subsubsection{Les cinq modes : «formule " distinctive et zone de formes.}

C'est chacun des modes qui - comme le DD pris à titre d'exemple - est appréhendable en langue aux deux niveaux :

(I) de sa « Formule » définitoire en traits pertinents ;

(II) de la zone de formes, diverses, selon lesquelles il peut se réaliser linéairement.

Ainsi, dans le système des trois traits A-B-C posé ci-dessus, au mode $\mathbf{D D}$, défini comme $\left[\mathbf{A}_{1}-\mathbf{B}_{2}-\mathbf{C}_{2}\right]$, répondent :

- le mode $\left[A_{1}-B_{1}-C_{1}\right] d u D I$ (discours indirect) : représentation de $\boldsymbol{a}^{\mathbf{0}}$ comme objet, via un fonctionnement sémiotique ordinaire et un ancrage énonciatif 
unique $^{23}$ (« normal », en A) ; la zone de formes répondant à ces propriétés comportant, notamment :

(6) (a) Il reconnaît qu'il s'est trompé/s'être trompé/son erreur. (b) Il a, reconnaît-il, des rapports difficiles avec ses voisins. (c) Il a dit ce qu'il avait à dire/expliqué comment faire. (d) Il a menacé ses voisins/posé des questions embarrassantes. (e) Ils ont évoqué leur jeunesse/ parlé affaires. Etc.

- le mode $\left[A_{2}-B_{1}-C_{1}\right]$ de la MAS, modalisation d'assertion comme seconde : représentation de $\boldsymbol{a}^{0}$ comme source de l'assertion énoncée, via un fonctionnement sémiotique ordinaire et un ancrage énonciatif unique (en A) ; la zone de formes répondant à ces propriétés comportant :

(7) (a) Selon lui/d'après lui/si on l'en croit, il a des chances de l'emporter. (b) Il paraît qu'il a des chances de l'emporter. (c) Il a, paraît-il, des chances de l'emporter. (d) Il aurait des chances de l'emporter. Etc.

- le mode $\left[A_{2}-B_{2}-C_{1}\right]$ de la MAE, modalisation autonymique d'emprunt : représentation de $\mathbf{a}^{0}$ comme source d'un segment de la chaîne énoncée, via un fonctionnement sémiotique avec autonymisation et un ancrage énonciatif unique (en A) ; la zone de formes répondant à ces propriétés comportant :

(8) (a) C'est un « marronnier», comme on dit dans la presse/pour parler comme dans la presse/ selon le jargon de la presse. (b) Il y a là ce que Althusser appelle/nomme une coupure épistémologique. (c) Le prétendu, fameux, sempiternel « déclin de l'occident », correspond... (d) La mère a été jugée « déméritante » (sic). Etc.

- le mode $\left[\boldsymbol{A}_{1}-\boldsymbol{B}_{2}-C_{3}\right]$ du Bivocal/DIL : représentation de $\boldsymbol{a}^{0}$ comme objet, avec autonymisation via le « parler avec » d'un partage des ancrages énonciatifs primaires entre $\mathbf{A}$ (référentiels) et $\boldsymbol{a}$ (modaux) ; la zone des formes correspondant à cette propriété, comportant :

(9) (a) Jean ${ }_{\mathrm{i}} \mathrm{s}^{\prime}$ informe : « Peut-il $\mathrm{l}_{\mathrm{i}}$ emprunter le livre ? ». (b) Jean ${ }_{\mathrm{i}}$ passe la tête. Peut-il $\mathrm{i}_{\mathrm{i}}$, demande$\mathrm{t}-\mathrm{il}_{\mathrm{i}}$, emprunter le livre ? (c) Jean ${ }_{\mathrm{i}}$ passe la tête. Peut-il $\mathrm{l}_{\mathrm{i}}$ emprunter le livre ? (d) Jean $\mathrm{j}_{\mathrm{i}} \operatorname{soupira}$ Eh voilà, $\mathrm{il}_{\mathrm{i}}$ n'aurait pas le livre indispensable! Etc.

23 On voit que, relativement à la variabilité des contours assignés au " discours narrativisé » lorsqu'il est distingué du DI, et aux positions différentes quant à une séparation/réunion du DI et du narrativisé ( $c f$. par exemple López Muñoz et al. 2004 : 19), la définition d'un Mode DI par la formule $\mathbf{A}_{\mathbf{1}}-\mathbf{B}_{\mathbf{1}}-\mathbf{C}_{\mathbf{1}}$ débouche sur la plus large des inclusions - limitée par la seule « condition de représentativité » évoquée chap. 2.2.1, p. 54 - à charge pour la description de cartographier cette riche variation interne, aux plans - syntaxique, sémantique, discursif - où elle se déploie. 


\section{Le double étage où se spécifient les modes : Formule/zone de formes}

La différence de statut entre les deux niveaux (I) et (II) sur lesquels s'étage, en langue, cette structuration, est essentielle.

- La reconnaissance d'unités (formes) de langue, mais non pas de « syntaxe» ou de " grammaire » au sens étroit du terme, conduit à s'écarter de formulations - soucieuses pourtant de distinctivité linguistique - telles que, chez M.-A. Mochet (1994 : 249), par exemple (pour DD, DI, D narrativisé) :

Pour « mettre en scène » ces actes verbaux, le locuteur dispose principalement de trois modalités syntaxiques qui lui permettent [...].

Et, bien entendu, l'ancrage définitoire des modes, en langue, mais en deçà du grammatical au sens séquentiel du terme, écarte, dans son principe même, toute « dérivabilité » morpho-syntaxique entre les modes ${ }^{24}$.

De même, cette structuration en « Formule/zone de formes » des cinq modes est étrangère à la perspective « prototypique » telle qu'elle est proposée par von Roncador (1988) par exemple, organisant la diversité des formes observables comme plus ou moins proches ou périphériques par rapport au centre d'une forme (de DD, de DI, ...) posée comme prototype. De même que le phonème n'est pas une réalisation prototypique mais une unité abstraite correspondant à une classe de sons, de même le « mode DD » par exemple ne correspond pas à « une forme » particulière de réalisation ; et le DD non marqué (ou DDL) par exemple est « autant » du DD que la forme, donnée comme canonique, du l dit : « ... ».

Le double niveau à travers lequel se structure le système des cinq modes de RDA - celui du jeu différentiel des Formules définitoires (I) et celui de l'éventail des types formels par lesquels celles-ci se linéarisent (II) - ne présente pas le même degré de stabilité. Qu'elle soit sociolinguistique ou diachronique, la variation est à l'œuvre au plan (II) de l'espace des formes offertes à la réalisation d'un mode : à titre d'exemple, il en va ainsi du DI « sans que », variété populaire contemporaine ${ }^{25}$, largement attesté dans le corpus Vincent-Dubois (1997 : 34 sq.) tels (outre chap. 2. (57) p. 58) :

(10) I(l) dit i(l) va faire et après i(l) fait pas [oral, 4-6-2009, à propos d'un époux rétif au bricolage].

24 Telle qu'elle persiste encore, par exemple, chez Riegel et al. (1994 : 597), assortie cependant de la réserve : "Cette analyse grammaticale ne rend pas compte de tous les phénomènes de discours rapporté $[. .]$.$» .$

25 Participant du phénomène plus large d'effacement du que ; $c f$. (13) ci-dessous, avec vouloir comme avec dire. 
(11) moi je trouve qu'il est elle est bien il me dit il est bien là haut hé bon [cité in Marnette (2005: 156)].

(12) Ils ont dit ils passeraient dans chaque maison pour vérifier les canalisations [oral 10-112015, après une réunion municipale de quartier].

(13) - Ouais, y'en a en heure de vie de classe ils ont dit vous charriez trop. Ils voudraient je le dise en conseil de classe [F. Bégaudeau Entre les murs, 2006 ].

ou du $D D$ «avec que $»^{26}$ qu'il soit fréquent comme dans l'ancienne langue ${ }^{27}$ :

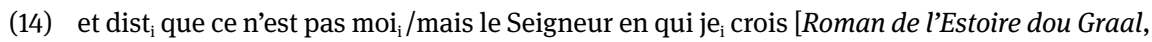
cité in Perret (1977 : 16) qui le reprend à Meiller (1966)].

ou nettement plus sporadique comme aujourd'hui ${ }^{28}$ :

(15) l'infirmière elle m'a dit que il faut que vous /partez, partiez/ avec l'ambulance /qui, il/ vous attend + elle m’a amené ici [cité in Marnette (2005: 156)].

(16) Gabriel $\mathrm{l}_{\mathrm{i}}[\ldots .$.$] rougit et se dit que cette fois, c'est sûr, \mathrm{je}_{\mathrm{i}}$ suis amoureux d'Ann [...] [Orsenna, L'exposition coloniale, 1988, cité in Charlent (1996)].

(17) Ma mère, elle est tout le temps après moi à râler, que tu penses qu'à t'amuser, que je travaille pas pour que t’ailles à l'école si tu fais rien, tout ça... [train de banlieue, mai 2008].

Ainsi le que n'apparaît-il comme ni nécessaire ni suffisant au DI : pleinement compatibles avec les « Formules » respectives des modes DI et DD (I), ces énoncés (10-17) s'inscrivent dans l'espace (II) des formes linguistiques dans lesquelles ces modes se réalisent ${ }^{29}$, appelant, au plan (III), une description de leurs conditions d’apparition en discours (écrit, oral ; littéraire, médiatique, populaire, enfantin...).

Et peut-être le débat sur la modernité ou au contraire l'ancienneté du DIL ${ }^{30}$ gagnerait-il à distinguer les deux plans (I) d'une Formule - stable - de traits, comportant celui $\left(\mathrm{C}_{3}\right)$ de la division des ancrages énonciatifs et (II) des types linéaires - sujets à variation - selon lesquels elle prend forme - depuis sa première attes-

26 Entendu ici - restrictivement par rapport aux conceptions assimilant MAE et DD critiquées ci-dessus (chap. 8.3.3 p. 304 et chap. 8.4, p. 308) - comme énoncé en $V$ de parole + que présentant une dualité d'ancrage énonciatif, d'abord en A puis en $\boldsymbol{a}$.

27 Cf. l'étude de Bruña Cuevas (1996) et la présentation de Rosier (1999 : 82-87).

28 D'un emploi « rare » aujourd'hui en français parlé, selon Marnette (2005: 176) qui en relève $0,3 \%$ dans son corpus.

29 Formes - diverses - qui sont à intégrer aux réflexions sur le statut du morphème que en français contemporain : $c f$., notamment, Delofeu (1999) pour les traitements proposés des emplois de que ne relevant pas de la « simple » subordination conjonctive ; et les remarques de de Gaulmyn (1983) sur le que, jonctif, pré-subordonnant, en langage enfantin.

30 Pour une présentation claire du débat, $c f$. Pernot (2008). 
tation littéraire ${ }^{31}$, dialogale, dans la brève réponse, dite ou pensée, de l'héroïque Eulalie aux menaces du roi païen, jusqu'aux émergences, dans le roman moderne, d'amples coulées de discours intérieur, non adressé, qui, si novatrices soient-elles, aux plans (II) et (III), ne mettent pas en cause l'identité du mode, telle qu'elle est définie au plan $\mathrm{I}$.

On voit ici - selon l'heureuse formule de Barthes (1977), soulignant que si « la langue afflue dans le discours, le discours reflue dans la langue » - l'incidence $\mathrm{du}$ fonctionnement discursif sur l'éventail des types de formes correspondant à un mode : ainsi, outre les transformations de la pratique narrative littéraire, le développement d'une écriture journalistique (de « titraille » par exemple), de la communication audio-visuelle, des techniques électroniques d'interaction (courriel, échanges sur " réseaux sociaux »...) sont-ils susceptibles d'induire de nouveaux types de formes de RDA ; mais, aisément observable au plan II des modes de RDA, ce « reflux » du discours dans la langue ne se manifeste pas au plan I de leur formule définitoire dont l'altération, touchant plus profondément à la structure de la langue, impliquerait d'autres mécanismes, inscrits sur une autre temporalité...

Dégager, comme on l'a fait, en deçà des énoncés et même des types de formes, une identité abstraite, différentielle, aux divers modes ainsi distingués, conduit à les reconnaître - non affectés encore des enjeux psychologiques, argumentatifs... dont ils se chargeront en discours - comme les alternatives que propose la langue, en nombre fini, pour l'appréhension d'un discours autre : le jeu différentiel des combinaisons de traits sémantiques (A : parler de/parler d'après), sémiotique (B : projeter ou non les signifiants sur le devant de la scène), énonciatif (C : intégrer/ disjoindre/partager l'ancrage énonciatif autre) est ce qui dessine, façonne les « perspectives tout à fait différentes face au discours d'autrui » qu'évoque G. Philippe (1996 : 142) à propos de l'opposition DD/DI.

S’il n'est pas question d'assimiler des approches relevant de cadres très différents à la conception des modes de RDA esquissée ici, on peut néanmoins faire apparaître entre elles des points de résonance. Ainsi, sans entrer dans la problématique complexe proposée par Bally (1914) de l'articulation entre « Figures de pensée et formes linguistiques » - et sans y assimiler le double niveau des

31 Dans la célèbre Cantilène de Sainte Eulalie (vers 880 ), où celle-ci à l'abjuration de sa foi préfère le martyre : « [Le roi paien] li enortet [...] / Qued elle fuiet lo nom christien. / Ell'en adunet lo suon element : / Melz sostendreiet les empedementz / Qu'elle perdesse sa virginitet. / Por os suret morte a grand honestet. [Adaptation (Ch. Oulmont, La poésie française du moyen âge Mercure de France 1913) : Le roi paien] l'engage, [...], /À abandonner le nom chrétien /Elle en rassemble les siennes forces /Elle supporterait les tortures/ Plutôt que de perdre sa virginité./ C'est pourquoi elle mourut très honorablement]. 
«Formules » de traits et des zones de formes posé ici - peut-on souligner chez lui, développé à propos du discours rapportée ${ }^{32}$, le souci de dégager un plan d'analyse et de réel langagier ${ }^{33}$ qui pour n'être pas " grammatical » n’en relève pas moins de la langue : tel est le point de vue qui s'exprime lorsqu'il renvoie les erreurs touchant au «style indirect libre » à la méconnaissance chez les grammairiens du fait :

[qu'il] n'est pas une forme de grammaire, c'est une attitude de l'esprit, un aspect, un angle particulier sous lequel il perçoit les choses ; et - chose à bien noter - ce n'est pas une observation psychologique qui fait découvrir cette forme de pensée, elle se déduit de l'étude même de la langue. (Bally, 1912 : 606).

Et on retrouve dans le couple «schéma/variantes ${ }^{34}$ que fait travailler Voloshinov (1929), dans la section consacrée au discours rapportée ${ }^{35}$, des caractéristiques du double niveau Formule/formes linéaires : à la question de "l'appréhension active du discours d'autrui » répondent, selon lui, dans une langue, à un moment donné, quelques « schémas stables » " constitués et effectifs en langue » (Voloshinov 1929 : 164), « formes autonomes » (ibid.:197) ${ }^{36}$ qui « expriment », chacun, « une tendance » dans « la dynamique réciproque des deux discours » :

Chaque schéma recrée à sa manière l'énonciation [autre], lui donnant ainsi une orientation particulière, spécifique. (ibid. : 176).

32 Envisagé comme « occasion de dégager, à propos d'un fait particulier, une question de linguistique générale : le rapport à établir entre les figures de pensée et les formes linguistiques et spécialement les formes grammaticales. » (Bally, 1914 : 407).

33 Un « sentiment intuitif de la langue » dit G. Philippe (1996:149) à propos des «figures de pensée ». Pour le positionnement de Bally dans les débats « fondateurs » du DIL, évoqués chap. 4 note 61, p. 139, cf. Philippe et al. (2018).

34 Traduit en «modèles/modifications » dans la traduction de (2015: 385 sq.).

35 Ici encore, sans entrer dans le détail (compliqué par les problèmes de traduction, $c f$. Sériot 2011) d'une approche du fait langagier revendiquée - en opposition à «l'objectivisme abstrait » de Saussure - comme « intégralement sociale », ni, par conséquent, dans les différences certaines que cela implique entre les « Formules » des modes proposées ici et les « schémas » de Voloshinov, postulés dans une logique d'incidence sociale quasi directe sur les formes de langue ( $c f$. le sous-titre de l'étude du discours rapporté : « Essai d'application de la méthode sociologique aux problèmes syntaxiques »; voir l'éclairante présentation qu'en donne Combettes (1989 : 114-116). 36 Cf. la discussion évoquée ci-dessus (chap. 4.3.1, p. 132) sur la reconnaissance du DIL comme « schéma » à part entière (et non mélange DD/DI) et son apparition comme celle d'une « nouvelle forme linguistique » « complètement autonome» (Voloshinov, 1929 : 196, 197). 
Le « rapport des variantes au schéma de base » est celui, relativement à la forme abstraite dont elles procèdent, de réalisations multiples situées « à la frontière de la grammaire et de la stylistique » et « où s'accumulent les changements au cours des siècles » :

Le schéma ne se réalise que sous la forme d'une variante particulière. (ibid. : 173)

et, d’autre part, pour un schéma donné - celui du discours direct par exemple - :

Il [...] possède une immense variété de réalisations nettement différentes. (ibid. : 184)

\section{Jalons pour un parcours descriptif des cinq modes}

De la structuration évoquée ci-dessus se dégagent quelques axes - programmatiques - à même d'orienter la description de chacun des modes qui, supposant l'étude systématique de leurs formes et de leur fonctionnement en discours, incluant ce qui tient à leur réalisation matérielle (canal écrit ou oral et variété de leurs « supports » respectifs ${ }^{37}$ ) requiert l'espace d'un autre ouvrage.

\subsection{Incidences des rapports différentiels entre modes : « familles » de modes}

Les modes sont chacun, par définition, différents les uns des autres, mais ils le sont différemment, deux à deux, selon le nombre et la nature des traits qu'ils partagent ou qui les distinguent.

Il en est ainsi notamment de la façon dont les modes s'opposent/se rassemblent quant au trait $\mathrm{C}$ - porteur des types d'altération que le discours autre imprime au Dire au plan essentiel de son ancrage énonciatif ${ }^{38}$. Les traits $C_{2}$ et $C_{3}$ ne caractérisent, chacun, qu'un seul mode, DD et Bivocal respectivement. Le trait $\mathrm{C}_{1}$ détermine, lui, une «famille » de trois modes énonciatifs intégrés à A (MAE,

37 De l'imprimé sur papier - livres, journaux, brochures, prospectus... - aux scriptions passant par les matériaux les plus diverrs - affiches, pancartes, banderolles, tags, tatouages... - et la « dématérialisation » hyperformatée des courriers électroniques, SMS, « chats » et autres résaux sociaux à haute densité de RDA ; et, pour la parole, de ses réalisations dans l'espace privé ou public, du tête à tête à l'assemblée, du direct « physique » à tout ce qui passe « en direct » ou non par un medium technique, téléphone, dictaphone, répondeur et, évidemment, radio, télévision... 38 Référentiel et modal, primaire, $c f$. chap. 4.1.3, p. 109. 
MAS, DI), c'est-à-dire d'énoncés de statut énonciatif ordinaire, que n'altère pas leur appartenance au champ de la RDA. ${ }^{39}$

Cette répartition suggère pour le parcours descriptif du champ un cheminement qui - rompant avec l'ordre canonique DD-DI-DIL - partirait du groupe $\mathrm{C}_{1}$ des modes « simples » au plan énonciatif, avant d'envisager le $\mathrm{DD}$, seul à présenter la complexité par juxtaposition sur la chaîne d'une dualité énonciative, et, enfin, le Bivocal, dans la radicale singularité de sa division énonciative.

La pertinence des rapports « d'apparentement » plus ou moins étroits entre les modes - de zéro à deux traits partagés - mérite d'être envisagée dans le fonctionnement des modes au plan des combinaisons - cumul, enchâssements, enchaînements - qu'ils admettent ou privilégient, comme à celui du degré de stabilité de leur opposition en discours - ambiguïté, indécidabilité, neutralisation (cf. ci-dessous 3.3).

Ainsi, à titre d'exemple, leur appartenance à la « famille $\mathrm{C}_{1}$ » évoquée ci-dessus, semble, pour les trois modes qui la composent, aller avec une combinabilité intraphrastique spécifique, telle que l'illustre, par exemple, ce DI incluant une MAS et une MAE :

(18) Il prétend que sa voisine à laquelle, d'après lui, il n'aurait pas dû faire confiance, lui a « foutu tout son courrier à la poubelle » ... ! [correspondance, 20-11-2004].

À l'intérieur de cette même famille des modes énonciativement intégrés, un seul trait ([A] parler de/parler d'après) oppose DI et MAS qui ont en commun les deux autres ([B] et $[C]$ : sémiotique et ancrage énonciatif « ordinaires ») ; de même qu'un seul trait ([B] : sémiotique ordinaire $v s$ avec monstration de mots) oppose MAS et MAE, qui partagent les deux autres ([A] et [C] : parler d'après, ancrage énonciatif unique) : aussi, reposant sur un seul trait, les oppositions entre ces modes apparaissent-elles structurellement propices, en cas d'effacement du marquage de ce trait à divers faits d'indétermination entre l'un et l'autre. Il n'en va évidemment pas de même pour une opposition maximale - à trois traits - comme celle qui sépare la MAS du DD, plus « résistante » à la neutralisation en discours.

39 Si on le restreint à son versant référentiel (déictique), le trait d'ancrage énonciatif (primaire) intégré, définit en compréhension un ensemble plus vaste que la « famille » $\mathrm{C}_{1}$, incluant le Bivocal : il correspond au complémentaire du DD dans le champ de la RDA, celui des Discours autres à Deixis intégrée, ou unifiée. Une telle structuration peut apparaitre pertinente au regard de certains fonctionnements syntaxiques : ainsi, par exemple, de la partition que pose F. Gachet (2012) entre incises en DD vs « dans le reste de la RDA ». 


\subsection{Le niveau de marquage : un paramètre de variation concernant l'ensemble des modes}

Le parcours de l'espace de variation, interne à chaque mode, celui (plan II) de la zone des types de formes selon lesquels se réalise sa « Formule » différentielle (I), est une part essentielle de la description de la RDA. Les paramètres qui y jouent de façon pertinente sont propres à chacun des modes - à leur spécificité sémantique, sémiotique, énonciative - et ne peuvent être appréhendés hors de la description précise de ceux-ci (que ne vise pas le présent ouvrage). Un axe de différenciation apparaît cependant transverse aux divers modes, celui (bien entendu à travers les formes propres à chaque mode) du niveau de marquage de la forme de RDA.

\subsubsection{Une variation interne à chaque mode}

Un traitement antérieur ${ }^{40}$ de cette dimension déployait sur trois degrés une opposition " explicite vs interprétatif », envisagée comme structurant le champ au même titre que les oppositions notées ici [A] et [B], et proposait une partition entre formes :

- marquées univoques : DD, DI, MAS, MAE ;

- marquées exigeant un travail interprétatif : guillemet ou italique marquant une MA devant être interprétée comme MAE ;

- purement interprétatives : DDL, DIL, citation cachée (allusion)...

De cette présentation, on peut retenir l'importance dans le champ de la RDA de la question du niveau de marquage et de sa variation, en rapport inverse avec la place conférée à la reconnaissance interprétative en discours ; et l'articulation des deux processus intervenant dans l'identification : celui du repérage univoque de marques isolables sur la chaîne (un selon $l$, des guillemets...) et celui de la mise en relation interprétative - de discordance et de concordance, notamment - d'une chaîne $\mathbf{e}$ en $\mathbf{E}$ avec l'ailleurs de son environnement intradiscursif, immédiat ou lointain, du contexte situationnel Sit de son énonciation $\mathbf{A}$, et de l'espace interdiscursif.

Mais la distinction des deux plans des modes - Formule définitoire et zone des formes par lesquelles ils se linéarisent - impose une remarque et deux déplacements de fond :

40 Dans un texte à visée pédagogique Authier-Revuz (1993a : 41-42) (structuration évoquée dans l'article « Discours rapporté » in DAD 2002). 
- la question du degré de marquage ne peut évidemment pas faire l'impasse sur la prise en compte, à l'oral, de l'accompagnement prosodique et mimo-gestuel à même d'assurer la reconnaissance univoque (comme DD ou MAE, par exemple) d'un segment dépourvu de tout marquage morpho-syntaxique, ou de marquer la borne finale du message représenté, notamment lorsque celui-ci, marqué morphosyntaxiquement à l'initiale (en DD, DI, MAS) est de nature pluriphrastique ${ }^{41}$. Par rapport au caractère binaire du marquage typographique du guillemet par exemple, la variation intonative et gestuelle susceptible d'indiquer une MAE est à la fois beaucoup plus riche - de degrés et de nuances - et moins sûre - ouvrant sur du «à peine marqué ».

- C'est au plan de la variation interne à chaque mode que doit être saisi le degré de marquage et non comme un trait qui les opposerait comme définitoirement marqués/non marqués. DDL et allusion ${ }^{42}$, par exemple, sont à traiter comme des formes, à marquage zéro, respectivement des modes DD et MAE, c'est-àdire comme des pôles de non-marquage sur l'échelle que parcourt leur espace de variation ${ }^{43}$;

- tous les modes présentent une variation dans le niveau de leur marquage : d'une part la variation qui parcourt la zone II de leurs réalisations linéaires atteint, sans exception, à une forme non-marquée, où - comme on l'a classiquement relevé pour le Bivocal-DIL - c'est de façon complétement interprétative qu'une chaîne en tant qu'énoncé, en discours, c'est-à-dire au plan (III), est reconnue comme réalisation d'un mode, répondant, à l'égal des formes marquées de la zone (II), à la Formule définitoire (I) du mode ${ }^{44}$.

41 Cf. ci-dessous (19) par exemple. Pour des éléments de repérage de la borne finale du message de DD voir notamment Morel et Danon-Boileau (1998 : 129-133) et Branca et al. (2012).

42 Cf. pour l'allusion, les exemples chap. 5 (1) p. 153, chap. 8 (88'), p. 299, et chap. 11 (1), (2), p. 427. 43 Suivant cette définition du DDL, on analysera (en accord avec L. Rosier $(1999$ : 279) excluant tout marquage typographique) comme DD faiblement marqué (non univoque) des énoncés comme " Jean marche vers la gare. "Je suis en retard" ", ou le titre du livre de A. Ernaux : "Je ne suis pas sortie de ma nuit » (et pas comme DDL, comme par exemple Romeral $(2005: 115,117)$ incluant dans le DDL les formes marquées typographiquement)

$44 C f$. ci-dessous chap. 11.2.1, p. 427 Sur cette question du marquage zéro, je renvoie à la lecture aiguë que R. Mahrer (2011 : 210-218 notamment) propose de la langue benvenistienne comme "système de signes informé par des fonctions » - fonctions " qu'assure dans le milieu humain » (Benveniste (1974 : 217) comme réponses données aux problèmes qui s’y posent, un langage qui primordialement « signifie ». Citant Benveniste selon lequel des « formes très dissemblables sont à classer ensemble au point de vue de leur fonction » et « les agencements matériels que constate et analyse la linguistique descriptive peuvent être progressivement ramenés aux figures diverses d'un même jeu » (souligné par R.M.), il envisage la "possibilité du marquage nul » - « soit de mobiliser "en discours" la valeur d'une fonction sans recourir à son appareil formel 
Ainsi, par exemple, le DI et la MAS connaissent-ils, eux aussi, des formes non marquées, qui relèvent interprétativement, notamment, de l'extension extraphrastique - à une ou plusieurs phrases - d'un marquage antérieur ${ }^{45}$.

En ce qui concerne la MAS, l'incidence au-delà de la phrase des selon $l$, pour l..., observable dans les énoncés ci-dessous, a été précisément étudiée ${ }^{46}$ dans la perspective de leur fonction « cadrative » reconnue par Charolles (1987) :

(19) D’après M. H., l'azalée, c'est pas une bonne idée. \{Même en ajoutant de la terre de bruyère, le sol est trop calcaire $\}_{2}$. \{ll a eu plusieurs échecs dans le coin $\}_{3}$. Il m’a conseillé d'autres arbustes plus accommodants [oral, nov. 2012].

(20) À en croire les croupiers, Aliso avait joué environ trois heures le jeudi soir, et pour finir était plus ou moins rentré dans ses sous. \{Le vendredi, il avait encore joué deux heures en début de soirée et d'après leurs estimations, avait quitté la table délesté de deux mille dollars $\}_{2}$ [M. Connelly, Le cadavre dans la Rolls, trad. fr. Points, 1998, chap. 2].

(21) Pour Chantal Crétaz, secrétaire général de l'Association Nationale des Visiteurs de Prison, la prison en France n'est «plus un lieu de justice ». \{Il en va de l'intérêt de tous de permettre à un détenu de garder sa dignité car, "faire du détenu-sujet un détenu citoyen », c'est participer à la préservation de la sécurité publique ${ }_{2}$ [Libération, 28-02-1997, p. 15, compte rendu d'un colloque sur les conditions de détention, idt] ;

En revanche, a moins été prise en compte l'extension, au delà de la complétive, de l'incidence de $l$ dit que $e^{47}$ telle que, très communément ${ }^{48}$, elle fonctionne :

propre » -, c'est-à-dire « qu'une identité puisse être reconnue [...] au-delà de l'opposition avec marques/sans marques » comme «l'une des manifestations les plus évidentes de la gouvernance du sens dans les procédures d'analyse » (Mahrer $2011: 215$ ).

45 Parmi les éléments jouant dans l'interprétation " par extension » d'une forme non marquée de DI ou de MAS, le genre du discours apparaît comme spécifiquement pertinent : ainsi est-ce la forme privilégiée de représentation des « tours de parole » dans nombre de genres - très normés - de discours « tenant lieu » d'un autre discours - tels que procès verbaux, comptes rendus, (cf. Mellet et Sitri 2013, Authier-Revuz et Lefèbvre 2015, Sitri 2015b), sur le mode : A dit que P. Q. R. ; B remarque que P. Q. R. ; C souligne que P. Q. R. ... Ce « patron », dominant dans le genre « procès-verbal » du rapport de soutenance de thèse, D. Maingueneau (in Dardy et al. 2002) en décrit pertinemment le fonctionnement (p. 73-86), mais dans les termes - à mon sens problématiques - d'un DIL de « type singulier », « très appauvri », dont « on a l'impression que [la] catégorisation se fait par défaut ».

$46 C f$. notamment Schrepfer (2006) tout entier consacré à la « portée » des selon l, dans un corpus du Monde Diplomatique.

47 Sa perception étant sans doute obscurcie par l'existence du DIL-Bivocal, parfois ramené à du DI « sans que », $c f$. chap. 4.3.3 note 64, p. 141. La question du « scope » d'un opérateur de discours rapporté est envisagée par Kerbrat-Orecchioni (1980 : 163), qui signale, en l'illustrant d'un exemple de DI (du type l a annoncé que P. Q.), que « dans certains cas l'incidence du verbe introducteur enjambe une frontière de phrase ».

$48 C f$. encore exemples chap. 6. (39), p. 219 et chap. 11. (4), p. 428. 
(22) M. X, en charge des bâtiments scolaires, signale que le groupe scolaire de $\mathrm{X}^{\star \star}$ requiert des travaux importants. \{Des infiltrations ont été constatées en plusieurs points $\}_{2}$. \{L'installation électrique de la partie ancienne n'est plus en conformité avec les normes actuelles $\}_{3}$. Il conclut qu'une réévaluation de la dotation initiale s’impose [Procès-verbal de réunion de conseil municipal (2000)].

(23) Kramer (1985) remarque que même si le décès de la génération plus âgée est prévisible, son impact est très puissant sur la famille. \{Les émotions sont toujours fortement intensifiées lorsque le système familial vit une perte $\}_{2}$. \{Avec la mort d'un membre, en particulier s'il s'agit d'une figure centrale, un vide est créé $\}_{3}$. Kramer pose dès lors les deux questions [...] [É. Goldbeter-Merifeld, Deuils et fantômes, p. 76].

(24) Derrida remarque que Lacan se sert de la littérature pour illustrer la vérité de sa doctrine, c'est-à-dire une vérité extérieure au texte littéraire. \{En agissant ainsi il rejoint la pratique de la psychanalyse appliquée alors même qu'il en condamne l'usage $\}_{2 .}$ Outre cette contradiction, Derrida souligne [...] [É. Roudinesco, Histoire de la psychanalyse en France, t. 2, Seuil, Paris, p. 545].

(25) Il dit qu'il va améliorer son service, peut-être adopter une raquette au manche rallongé pour lui donner un surplus de puissance. \{Poursuivre le double aussi, qui l'a poussé vers un tennis plus audacieux, plus offensif $\}_{2}$. $\{\text { Il va jouer à Prague, disputer les qualifs à Rome }\}_{3}$. \{Et pointer ce tennis aux contours étranges du côté de Roland-Garros $\}_{4}$. Il jure: «Je joue vraiment au tennis aujourd'hui. » [Libération, 28-04-1997].

(26) Il lui $i_{i}$ fut dit que non seulement la petite Fadette n'y était point venue enceinte et n'y avait point fait d'enfant, mais encore qu'elle s'y était si bien comportée qu'il n'y avait point le plus petit blâme à lui donner. \{Elle avait servi une vieille religieuse noble, laquelle avait pris plaisir à en faire sa société plus que sa domestique, tant elle l'avait trouvée de bonne conduite, de bonnes mœurs et de bon raisonnement $\}_{2}$. \{Elle la regrettait beaucoup, et disait que c'était une parfaite chrétienne, courageuse, économe, propre, soigneuse, et d'un si aimable caractère, qu'elle n'en retrouverait jamais une pareille $\}_{3}$. [...].

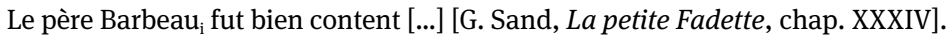

Et, à l'inverse, le mode Bivocal est bien un mode " interprétatif ", en ce que, contrairement aux autres modes, il ne présente pas de forme phrastique univoque hors contexte, d'où la nécessité de recourir, pour les exemples fabriqués, à des indices stipulant les co-références requises pour une interprétation en Bivocal

(27) $\mathrm{Jean}_{\mathrm{i}}$ reprend son travail. Marie va-t-elle $\mathrm{l}_{\mathrm{i}}$ 'appeler?

là où cette spécification interprétative est le plus souvent superflue pour les exemples empruntés à des textes, c'est-à-dire pourvus de contexte. Mais le Bivocal se réalise aussi dans des formes comme ${ }^{49}$ :

(28) $\mathrm{Jean}_{\mathrm{i}}$ proteste : «Pourquoi, s’exclame-t-i $\mathrm{l}_{\mathrm{i}}$, ne lui $\mathrm{i}_{\mathrm{i}} \mathrm{a}$-t-on pas dit de venir ? »

49 Cf. chap. 4.3 .1 note 46, p. 131. 
où c'est une forme marquée à un fort degré comme $R D A$ qui est à interpréter comme Bivocal ${ }^{50}$.

\subsubsection{Marquages partiels et marquages « retardés "}

Le marquage - ou son défaut - n'est donc pas à penser comme assurant ou non « en bloc » l'identification d'une séquence comme forme d'un mode déterminé de RDA. Des marques univoques, jouant dans le champ de la RDA, se situent en retrait par rapport à l'identification d'un mode, ouvrant sur un travail interprétatif d'assignation à l'un de ceux-ci ou sur un fait de neutralisation d'opposition. Ainsi, une séquence marquée de la dimension autonymique par des guillemets ne serat-elle (ou non) identifiée, d'abord comme RDA, ensuite comme relevant du mode DD, MAE ou Bivocal, que combinée à d'éventuelles autres marques ou éléments discursifs d'interprétation.

Le statut de l'incise de type dit-il est de ce point de vue exemplaire : si elle constitue, de fait, une marque univoque, c'est celle de l'appartenance à la RDA de la séquence qu'elle commente - par opposition au type dis-je, au présent, de l'ARD - ; et non, par elle-même d'un mode spécifique de RDA : c'est par le jeu combiné des autres marques et/ou indices interprétatifs de l'énoncé où elle figure, qu'aboutira, ou non, l'identification d'un mode particulier.

Il en est ainsi dans les exemples suivants, manifestant la compatibilité de dit-il avec tous les modes, l'incise ne constituant pas, par conséquent, une « marque » de DD :

$\begin{array}{ll}\text { (29) a DD } & \text { Marie }_{\mathrm{i}} \text { était furieuse : «Pour qui, dit-elle } e_{i}, \mathrm{me}_{\mathrm{i}} \text { prend-on ? » } \\ \text { b Bivocal } & \text { Marie }_{\mathrm{i}} \text { était furieuse : «Pour qui, dit-elle } \\ \text {, } \text { la }_{\mathrm{i}} \text { prenait-on ? » } \\ \text { c DI/MAS } & \text { Marie est allée, dit-elle, à plusieurs expositions. } \\ \text { d MAE } & \text { Marie est allée à plusieurs expositions, «fascinantes », dit-elle. }\end{array}$

Comme, par exemple, dans :

(30) Bivocal

Elle $_{\mathrm{i}}$ n'en voulait point à sa mère, elle se reprochait seulement de ressentir moins d'amour que de terreur pour elle. Peut-être, pensait cet ange ${ }_{i}$ ces sévérités étaient-elles nécessaires ? Ne l'avaient-elles pas préparée à sa $\mathrm{i}_{\mathrm{i}}$ vie actuelle? En l'écoutant, il me semblait [...] [Balzac, Le Lys dans la vallée, p. 95].

50 Plutôt que comme DD ( $c f$. (29a) Jean ${ }_{\mathrm{i}}$ proteste : « Pourquoi, s'exclame-t-il $\mathrm{i}_{\mathrm{i}}$, ne lui $\mathrm{i}_{\mathrm{j}}$ a-t-on pas dit de venir ?») ce qui montre qu'une partie des exemples proposés comme formes de DD (fabriqués, donc hors-contexte discursif) ne relèvent pas du seul « marquage » univoque, mais aussi d'une interprétation qui, spontanément majoritaire, passe aisément inaperçue. En toute rigueur, mais au risque d'alourdir, il aurait fallu « indicer » ces exemples incomplètement marqués de DD hors contexte, comme les exemples de Bivocal. 
(31) DI/MAS Quatre heures dans les phrases, ce n'est pas mal. Mais le vieux Casanova $\mathrm{i}_{\mathrm{i}}$, en Bohème, écrivait, dit-il , douze à treize heures par jour [Ph. Sollers, Dictionnaire amoureux de Venise, 2004].

(32) MAE Qu'en est-il des biographes des grands poètes, des grands artistes, des grands penseurs, des « Mages », disait Victor Hugo ? [J. Bruneau, Préface au Gustave Flaubert de H. Lotman].

Outre la neutralisation opérée par l'incise quant au trait source/objet ${ }^{51}$ qui distingue notamment les modes énonciativement intégrés et sans autonymie $\left(\mathrm{C}_{1}\right.$, $B_{1}$ ), DI et MAS, nombreux sont les énoncés, univoquement marqués comme RDA par l'incise, que leur forme ne spécifie pas dans l'ensemble MAS-DI-Bivocal, par exemple :

(33) Gandhi $\mathrm{i}_{\mathrm{i}}$ se boucha les oreilles, mais refusa que l'on éteigne la lumière de son compartiment. Les gens devaient le voir si tel était leur souhait, dit-il $l_{i}$ : malgré le vacarme, il parvint à écrire. [...] fin octobre 1946, $\mathrm{il}_{\mathrm{j}}$ pressa Gandhi $\mathrm{i}_{\mathrm{i}}$ de repousser son départ ; Calcutta, disait-il ${ }_{j}$, avait aussi besoin de lui [R. Gandhi, Gandhi, trad. française 2008, p. 731].

ou, au-delà, incluant le DD :

(34) Il faut dire que les parents en redemandent : associer les enfants aux achats familiaux, c'est, disent-ils, leur apprendre la vie tout en achetant utile [Le Nouvel Observateur, n ${ }^{\circ} 1923$ ].

Rappelons la problématique, distincte, et valant y compris pour des formes univoquement marquées comme relevant d'un mode donné, de la délimitation du e représenté entre ses deux bornes gauche et droite, dans laquelle se combinent (différemment à l'oral et à l'écrit) marques et indices démarcatifs et indices discursifs de cohérence/disjonction.

Le « jeu » dans le marquage de la RDA relève aussi, dynamique, de son inscription dans le mouvement $d u$ dire : au-delà des variations intraphrastiques de déplacement d'un dit $\boldsymbol{l}$ ou d'un selon $\boldsymbol{l}$ en tête ou au contraire en fin de phrase, riches en effets de sens, il faut noter, plus radical, le processus interphrastique de marquage du type

P. C'est $\boldsymbol{l}$ qui le dit.

51 Cf., signalés au chap. 3, p. 101, les faits de neutralisation DD/MAE (ex. (62) et (63)) et DI/ MAS (ex. (65)) avec incise en dit-il. Voir aussi Komur (2003: $266 s q$.) qui relève dans la presse, pertinemment analysés comme " archi-formes » de RDA, des faits de neutralisation de formes de RDA : DD-DI-DIL avec incise, et les réflexions de Nita \& Hanote (2007) sur le caractère de « discours rapporté indéterminé » relativement aux « formes classiques de DR », de formes avec incise de parole. 
qui, à une phrase non-marquée fait succéder, avec des effets divers, son marquage univoque rétroactif ${ }^{52}$ :

(35) Ce n'est même pas lui qui a choisi sa femme, alors rendez-vous compte. Enfin c'est ce qu'on dit [F. Vargas, L'armée furieuse (2011), p. 358].

(36) Ici le jour ne ressemble pas au jour. Le jour est comme un morceau délavé de la nuit. L’accompagnateur, par ces mots, veut gifler les esprits [Incipit de L. Salvayre Les Belles âmes, 2001].

(37) Pauvre Jeanne! Pauvre Emma!

Pauvres femmes! Quoique, après tout, si elles étaient un peu moins bêtes, elles seraient peut-être un peu moins malheureuses (c'est Maupassant qui parle, ici, non l'auteur de cette préface) [A. Fermigier, Préface à Une Vie, Folio, p. 3].

Le mouvement rétroactif, ici, à partir d'une marque univoque, peut bien entendu fonctionner à partir d'indices interprétatifs.

Signalant la fréquence de ce procédé « d'éclaircissement rétroactif » dans le roman sartrien, G. Philippe (1997 : 48) renvoie à l'étude que P. Hamon ${ }^{53}$, sous le nom d' "épigraphie », propose des procédés qui consistent à attribuer un énoncé implicitement et a posteriori à un personnage en écrivant à côté « que l'énoncé qui précédait n'était pas attribuable au narrateur. »

Entre la mobilité intraphrastique (incises, constituants marqueurs de MAS) et les liens interphrastiques (anaphoriques...), s'ouvre l'ensemble des formes phrastiques « décrochées », en incidente, entre parenthèses, en note, enrichissant pour une phrase la gamme de ses marquages "seconds » (seconds dans le temps, ou la hiérarchie syntactico-énonciative, par rapport aux constructions du type $\boldsymbol{l}$ a dit $q u e . .$.$) et la panoplie des effets auxquels ils se prêtent.$

$\mathrm{Au}$ total, c'est non seulement loin d'une opposition binaire mais même d'une « échelle » totalement ordonnée ${ }^{54}$ que se réalise le marquage des diverses formes de RDA, combinant, de façon complexe, avec leur graduation propre, divers éléments : appartenance au champ de la RDA, identification d'un mode spécifique de RDA, tracé des frontières de l'énoncé $\boldsymbol{e}$ représenté, attribution à une source $\boldsymbol{l}$.

52 Rencontré chap. 3.1.2.1 ex. (35-38), p. 90 et $c f$. par exemple, pour le DD à l'oral, Morel et Danon-Boileau (1998 : 129-133) ou, pour la MAE, Authier-Revuz (2012 : 272 sq.).

53 Dans Textes et Idéologies, PUF (1984: 141).

$54 \mathrm{Au}$ sens de l'ordre total mathématique où tout point peut être situé par rapport à un autre sur une échelle linéaire - d'où une hésitation sur l'emploi du terme "degré » à laquelle son remplacement par « niveau » ne répond guère, pour cette structure " pluri-scalaire », de façon vraiment satisfaisante... 
C'est, par là, dans ce jeu pluri-scalaire, un espace considérable qui s'ouvre pour le marquage partiel des formes de RDA, auquel répondent deux aspects notables $\mathrm{du}$ fonctionnement de la RDA.

Le premier est celui de la part, souvent minimisée, du travail interprétatif qui, étroitement solidaire du degré de marquage, doit être soulignée dans l'identification de formes semi-marquées de RDA, contre l'assimilation de cas d'interprétation « évidente » à des faits de marquage univoque ${ }^{55}$ : c'est le cas, par exemple, pour la forme - massivement présente dans la presse ou le récit romanesque - du « $\boldsymbol{e}$ » de DD annoncé par ce qui constitue une phrase de DI catégorisant, du type :

(38) Il proteste : «e »

Contrairement à la contrainte syntaxique qui lie un « $\boldsymbol{e}$ » à son « introducteur » (recteur) dans la forme pleinement marquée il dit : « $\boldsymbol{e} »^{56}$, c'est un rapport de cohérence sémantique qui conduit, ou non, à interpréter en (39) la succession du DI et du « $\boldsymbol{e}$ » comme relevant de la représentation du même $\boldsymbol{a}^{0}$, la première forme de RDA « annonçant » la seconde, qui la « développe » en y trouvant son « $\boldsymbol{l}$ »; cette dimension interprétative, aisément dissimulée dans l'immédiateté de son fonctionnement, apparaît si on compare ces deux enchaînements de « $\boldsymbol{e}$ » à un DI représentant par catégorisation un acte d'énonciation, respectivement interprétables comme (a) développement de cet acte dont le DI est l'« annonceur »vs (b) « $\boldsymbol{e} »$ d'un acte distinct :

(39) Depuis le départ du train elle harcèle
la fillette :
$\begin{array}{ll}\text { (b) « Tiens-toi droite ! Ne t'agite pas comme ça ! » } \\ \end{array}$

Le second aspect à noter dans le fonctionnement de la RDA est celui, dans la diversité des types de « contacts » entre modes évoqués ci-dessous, des faits, solidaires de ces faits de marquage partiel, de « concurrence » de modes en un point de la chaîne.

55 C'est-à-dire ramenant ce que l'on « comprend » comme fait de discours, à ce qu'on « reconnaît » comme forme de langue, pour reprendre l'explicitation par Benveniste de l'articulation sémantique/sémiotique.

$56 C f$. chap. 8 (42) vs (41), p. 260 : il s'est fâché/il a lancé : «j'en ai assez ». 


\subsection{Contacts entre modes}

Entités différentielles, les modes sont distincts, mais non pas « séparés » en discours, présentant une variété de faits de « rencontre » d'un mode avec un autre.

\subsubsection{Concurrence de modes en un point de la chaîne}

C'est d'abord, en l'absence d'un marquage spécificateur d'un mode, la rencontre, « à la verticale » si l'on peut dire, en un point de la chaîne, de plusieurs modes, « candidats » à figurer en ce point, qui se résout selon les configurations de :

- l'ambiguïté, lorsque le contexte exige une assignation interprétative à l'un ou à l'autre, incompatibles, comme par exemple pour (28) dont l'interprétation doit trancher (en termes de coréférence) entre DD et Bivocal ;

- l'indécidabilité, lorsque rien ne permet, contextuellement, de trancher entre l'un et l'autre mode, entre lesquels oscille, instable, l'interprétation - comme souvent, par exemple, pour un « X » phrastique, syntaxiquement autonome, entre MAE ((je dis) « $X »$, comme dit $\boldsymbol{l})$ et DD (l dit : « $X »)$, tels, rencontrés précédemment (chap. 3 (62) ; chap. 8 (88')), les aphorismes « oignez vilain... » ou " la femme est l'avenir de l'homme ${ }^{57}$;

- la neutralisation d'opposition, lorsque l'identification, par marquage et interprétation, se situe au plan de ce que ces modes ont en commun, le sens se produisant en deçà de ce qui les distingue, comme on l'a évoqué, par exemple, ci-dessus avec l'incise en (33) et $(34)^{58}$.

Ainsi, la description du fonctionnement des modes, en discours, demande-t-elle que soient repérées les conditions d'apparition de ces cas de concurrence en un point, de plusieurs modes et de leur résolution en choix de l'un ou bien de l'autre

57 À propos des reprises (à incidences judiciaires) du « Casse-toi pov'con » initial de N. Sarkozy, alors chef de l'État, C. Mellet et F. Sitri (2012) dégagent, notamment p. 115, la double interprétation en DD : Sarkozy a dit : «Casse-toi... » et en MAE : «Casse-toi... », comme a dit Sarkozy.

58 Ainsi Marnette (2005 : 155 sq.) fait-elle place à une catégorie de « Discours Neutre » par rapport à l'opposition DD/DI : la possibilité de DI sans que (cf. (10-13) ci-dessus) en français parlé produit, en l'absence d'éléments déictiques ancrés en a ou en $\mathbf{A}$ et de marquage intonatif différenciateur, des énoncés tels : « et alors vous avez dit oh c'est très vrai tout ce que vous dites mais il y a une chose qui m'a frappée et vous dites elle ne pensait plus en français » (ibid: 156). Pertinemment, l'analyse de l'auteur va dans le sens, non de l'ambiguïté ou de l'indécidabilité, mais de l'en deçà de la neutralisation : « [...] it is doubtful whether the speaker wants or needs to make a difference here. What is important is the content of the reported discourse and indeed most examples of Neutral Discourse are used in an argumentative context [...] (ibid: 157). 
de l'ambiguïté, en oscillation entre l'un et l'autre de l'indécidabilité, en retrait en deçà de l'un et de l'autre de la neutralisation.

\subsubsection{Conjonction de modes}

La conjonction, sur la chaîne, d'un mode et d'un autre passe aussi par plusieurs configurations - phrastiques et interphrastiques - dont le fonctionnement mérite la description :

- cumul de l'un plus l'autre, tel que, par exemple, le présentent les constructions DI ou MAS avec MAE - $l$ dit que/selon $l$... « $X » . .$. - associant des modes à ancrage énonciatif intégré en $\mathbf{A}$ (famille $\mathrm{C}_{1}$ ).

Ces formes régulières au plan de la langue, sont stéréotypiques à celui du discours, par exemple dans le cas d'une MAE à statut d'îlot textuel ${ }^{59}$. Il importe de distinguer ces constructions régulières de cumul de deux modes, des mouvements intraphrastiques de « bascule » énonciative, d'un mode dans un autre, relevant d'une dynamique transgressive - par exemple d'un DI ou MAS vers un DD, ou, plus précisément, de l'émergence d'ancrages énonciatifs primaires, référentiels et/ou modaux, en $\boldsymbol{a}$, dans un énoncé intégré en $\mathbf{A}^{60}$. Il ne s'agit pas de séparer le bon grain (des cumuls réguliers) de l'ivraie (des phénomènes de rupture syntactico-énonciative), mais de reconnaître à ces derniers une hétérogénéité spécifique dont les ressorts méritent d'être interrogés. Que cette interrogation se fasse en termes de psycho-linguistique (défaillance, reprogrammation...), d'inscription subjective ou d'effet stylistique (de surgissement spontané ou concerté de la « voix de $\boldsymbol{l}$ » forçant le cours régulier du dire de $\mathbf{L}$ ), de diachronie (émergence d'une forme...), elle suppose, en tout état de cause, que ces phénomènes, saillants, de « bascule » énonciative, auxquels elle s'attache, ne soient pas, sous une rubrique, indifférenciante, de « formes mixtes/hybrides », confondus avec - c'est-à-dire noyés dans - la masse « sans histoire » des cumuls réguliers.

59 C'est-à-dire où il y a identité des $\boldsymbol{l}$ impliqués dans cette MAE et dans la forme DI ou MAS englo-

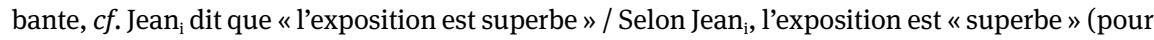
reprendre ses $\mathrm{s}_{\mathrm{i}}$ mots). (chap. 7.2.2.3 (40-41), p. 242, voir chap. 8.3.3.2 (89-94), p. 300.

60 Cf. chap. 8 (124) p. $309: \boldsymbol{l}_{i}$ évoque « comment ma $a_{i}$ vie tient à un fil », ou chap. 8 (109-111), p. 305, chap. 4 (40) et note 17, p. 110 et 116. Le fait de reconnaître, ou non, la MAE comme mode de RDA à part entière, distinct du DD (cf. 8.3.3.1, p. 297), joue évidemment un rôle crucial dans la distinction entre cumul et « bascule» (cf. 8.3.3.3, p. 305 ; ou 8.4, p. 309, par exemple, à propos de ce que recouvre problématiquement l'appellation « DD avec que »). 
- enchâssement, de l'un dans l'autre, correspondant à la représentation d'une chaîne d'actes d'énonciation ${ }^{61}: \mathbf{E}(\mathbf{A})$ représentant $\boldsymbol{a}_{\mathbf{1}}$ représentant $\boldsymbol{a}_{\mathbf{2}}$ représentant $\boldsymbol{a}_{3} \ldots$ sur le mode, par exemple, de $\boldsymbol{l}_{1}$ a dit que $\boldsymbol{l}_{2}$ lui avait dit que $\boldsymbol{l}_{3}$ disait... dont la description doit prendre en compte deux distinctions : d'une part celle qui se pose pour toute structure récursive entre grammaticalité en langue et acceptabilité en discours ; d'autre part, entre récursivité de $R D A$, via des combinaisons de modes divers, et récursivité d'un mode de RDA. En ce qui concerne la récursivité " à mode constant », les différences observables entre les divers modes quant à leur " aptitude » à l'auto-étagement relèvent de la mise en évidence des spécificités de chacun d'entre eux : on a noté (chap. 4.2.2) la disparité, en termes d'acceptabilité, de la récursivité du DI et du DD, aisée pour l'un, rapidement bloquée pour l'autre, le contraste renvoyant, évidemment, à l'opposition - aux plans syntaxique, énonciatif, sémiotique - entre l'unité du premier et la dualité du second ; de même, à la singularité énonciative - de division $\left(C_{3}\right)$ - du mode Bivocal-DIL dans l'ensemble de la RDA, fait écho une résistance, non moins singulière ${ }^{62}$, à la récursivité, que dégage G. Philippe (2005) en réponse à la question « Peut-on avoir du discours indirect libre dans du discours indirect libre? ».

- enchaînement de l'un puis de l'autre, présentées par des successions immédiates intra ou interphrastiques de deux (ou plusieurs) formes de RDA, syntaxiquement autonomes ; la fréquence de certains enchaînements dans ces " séquences composées " $^{63}$ de RDA - telles les successions DI-DD, ou « D narrativisé »-DD, étudiées par M.A. Mochet (1993) dans un important corpus d'entretiens oraux - fait apparaître des «patrons » différents, selon qu'ils correspondent à la représentation d'un seul acte $\boldsymbol{a}$, envisagé successivement sous deux angles, ou bien à la représentation de plusieurs actes distincts.

Ainsi, par exemple, pour des actes distincts, des effets de différenciation des deux voix en dialogue :

(40) Elle lui propose d’aller au cinéma. « Je n’aime pas ça. »

Elle suggère une promenade. «Je suis fatigué ».

Elle lui demande ce qu'il veut faire. « Rien ».

$61 C f$. Rosier (2005), (2006), traitant de la « circulation des discours ».

62 MAS et MAE n'interdisent nullement l'enchâssement, très généralement limité à deux étages : $\ldots$ « $X »$ comme dit $\boldsymbol{l}_{1}$ en reprenant $\boldsymbol{l}_{2} \ldots$; d'après $\boldsymbol{l}_{1}$, il paraît ${ }_{2}$ que les jeux sont faits.

63 Selon l'heureuse expression de M.A. Mochet (1994: 254). Cf. aussi les analyses de J. Bres (1996 : 56-58) montrant, dans des récits oraux d'interaction, les effets de " perspective » crées par les « alternances » séparant et hiérarchisant un arrière-plan au DI (et narrativisé) d'un $\boldsymbol{l}_{1}$ sur lequel se détache la saillance du DD d'un $\boldsymbol{l}_{2}$ en « je » (identifié au $\mathbf{L}$ narrateur). 
ou des enchaînements opposant - avec les visées les plus diverses - $\boldsymbol{l}_{1}$ dit que..., la formulation en usage d'un contenu, à $\boldsymbol{l}_{1}$ dit : « ... », la présentation de mots, en mention :

(41) X exprime sa confiance dans la réussite de l'entreprise. Y dit : « On court à la catastrophe ».

Et, pour un même acte, la succession, comme en (38) et (39a), du type :

(42) Il refuse : «Il n'en est pas question.»

(43) Il admet son erreur : « J'aurais dû lui en parler avant. »

suite stéréotypique au point de faire, parfois, méconnaître, sous l'appellation imprécise d' " introducteur de DD », la différence entre le lien - sémantique d'enchaînement entre les deux formes de RDA (l'une « annonçant » ce que l'autre « développe ») et le rapport syntaxique de rection, interne à une forme de RDA (il dit...) ; ou apparentable à ce que Voloshinov appelle « discours direct vidé de sa substance ${ }^{64}$, ce couplage DI-DD qui, faisant précéder les mots d'un $\boldsymbol{l}$ d'une formulation, en DI, par L qui en explicite - révèle, épuise - la charge sémantique, relègue le DD qui suit à une fonction d'accompagnement pittoresque, amusé, attendri, comme dans :

(44) On a joué à la balle, puis il m’a dit qu'il en avait assez : « Ça fussit, la balle, ça fussit » et on est rentré. [corresp. privée, juillet 1975, $\boldsymbol{l}$ enfant de 3 ans]

Cette RDA « double face » pour un même $\boldsymbol{a}$ apparaît aisément, dans les textes de sciences humaines ou de journalisme d'analyse de la société, comme « solution » à la difficulté d'un discours ayant pour « objet » des sujets parlants, solution aussi aisée qu'elle est redoutable par le statut « décoratif » de « couleur locale » qui y échoit aux mots de l'autre-objet au sein du discours, surplombant, du scripteur ${ }^{65}$.

64 Voloshinov (1929 trad. 1977 : 185), $c f$. aussi : « Le poids sémantique des paroles rapportées [au DD] diminue, mais en revanche leur signification caractérologique se renforce, ainsi que leur tonalité ou leur valeurs typiques » (p. 186) ; ailleurs (p. 169) Voloshinov évoque le processus de paroles qui, ayant perdu « leur sens objectif deviennent des objets décoratifs au même titre que le costume, l'aspect extérieur, les éléments constituant un tableau de mœurs, etc. »

65 Je renvoie aux analyses de B. Lagadec (1996 : 139) des formes de RDA dans les textes de P. Bourdieu introductifs aux entretiens reproduits dans La Misère du Monde, pointant le fait que les couplages DI du sociologue/DD d'un « jeune » qu'on y a observé « ne peuvent qu'aboutir à cette fameuse stigmatisation dénoncée par Bourdieu », et au travail d’A. Bolón (1996:196-207 notamment) sur des articles du journal Le Monde consacrés aux « jeunes issus de l'immigration », qui analyse les mécanismes de RDA (parmi lesquels les « $\boldsymbol{e}$ » de « jeunes » au DD succédant immédiatement à des DI, ayant déjà énoncé, dans les termes de $\mathbf{L}$ et de ses lecteurs, le sens des propos de $\boldsymbol{l}$ ) comme aboutissant, en dépit de l'abondance des mots « montrés » à la création d'une « absence de lieu de parole » (p. 196) pour des propos « dévitalisés ». Voir ci-dessous chap. 15, p. 616. 
Dégager les mises en œuvre dominantes, voire stéréotypiques, dans le discours de tel ou tel type d'enchaînement de formes de RDA, peut faire apparaître - aux plans générique, stylistique, idéologique... - des modèles de progression narrative, rhétorique, argumentative locale.

La structuration ainsi posée du champ de la RDA en cinq modes définis au double niveau d'une Formule (I) de traits distinctifs et de la zone de formes (II) par lesquelles la première peut se linéariser, ancre la RDA dans la langue comme système de différences. S'attacher à ce réel - abstrait - de distinctivité n'est pas délaisser le plan du discours : on a vu que l'inventaire (II) des types de formes propre à chaque mode (I) ne pouvait s'achever - renonçant par là à la complétude - sans faire place à la reconnaissance interprétative, en discours, des formes non ou semi-marquées de RDA ; et l'exploration systématique de chacun de ces ensembles (II) comme espaces de variation interne à chaque mode, parcouru d'un réseau propre de fines différences (construction, lexique, ponctuation, prosodie, pondération entre marquage de langue et indices interprétatifs, etc.) ${ }^{66}$ apparaît comme un préalable pour envisager, au plan de la foisonnante concrétude des énoncés (III) et de leur sens en discours, la part qu'y prend le jeu sur le clavier de la RDA - avec ses modes, leur Formule (I) et leurs formes (II).

Mais si rendre compte des faits de RDA observables en discours suppose d'en passer, en deçà de la concrétude des énoncés, par le réel abstrait de la langue et de ses différences, qui en sous-tendent le fonctionnement, il est aussi nécessaire, pour ces énoncés qui font place à du discours autre en le représentant, de prendre en compte cet autre réel qu'est, en tout énoncé, la présence constitutive - interdiscursive, dialogique - de la discursivité « extérieure ». À profiler les énoncés de RDA sur le fond de discours autre d'où ils émanent, s'impose la question pour le dire de la RDA, de la place dans l'économie langagière - subjective, discursive, textuelle - de ce geste énonciatif qui trace des frontières entre l'un et l'autre (représenté) dans un discours constitutivement traversé par de l'autre (présent). C’est ce à quoi s'attache la suite - partie V - du parcours.

66 Programme descriptif sur lequel débouche cette structuration, mais qui n'est pas l'objet du présent ouvrage. 